\title{
Instabilities of spin-liquid states in a quantum kagome antiferromagnet
}

\author{
M. Gomilšek, ${ }^{1}$ M. Klanjšek, ${ }^{1}$ M. Pregelj, ${ }^{1}$ F. C. Coomer, ${ }^{2}$ H. Luetkens, ${ }^{3}$ O. Zaharko, ${ }^{4}$ T. Fennell, ${ }^{4}$ \\ Y. Li, ${ }^{5}$ Q. M. Zhang, ${ }^{5}$ and A. Zorko ${ }^{1, *}$ \\ ${ }^{1}$ Jožef Stefan Institute, Jamova c. 39, SI-1000 Ljubljana, Slovenia \\ ${ }^{2}$ ISIS Facility, Rutherford Appleton Laboratory, Chilton, Didcot, Oxon OX11 OQX, United Kingdom \\ ${ }^{3}$ Laboratory for Muon Spin Spectroscopy, Paul Scherrer Institute, CH-5232 Villigen PSI, Switzerland \\ ${ }^{4}$ Laboratory for Neutron Scattering and Imaging, Paul Scherrer Institute, CH-5232 Villigen PSI, Switzerland \\ ${ }^{5}$ Department of Physics, Renmin University of China, Beijing 100872, People's Republic of China \\ (Received 22 October 2015; revised manuscript received 15 January 2016; published 16 February 2016)
}

\begin{abstract}
The emergent behavior of spin liquids that are born out of geometrical frustration makes them an intriguing state of matter. We show that in the quantum kagome antiferromagnet $\mathrm{ZnCu}_{3}(\mathrm{OH})_{6} \mathrm{SO}_{4}$ several different correlated yet fluctuating states exist. By combining complementary local-probe techniques with neutron scattering, we discover a crossover from a critical regime into a gapless spin-liquid phase with decreasing temperature. An additional unconventional instability of the latter phase leads to a second, distinct spin-liquid state that is stabilized at the lowest temperatures. We advance such complex behavior as a feature common to different frustrated quantum magnets.
\end{abstract}

DOI: 10.1103/PhysRevB.93.060405

In recent years, the intense search for quantum spin liquids (SLs) - collective highly entangled states in which long-range magnetic order is suppressed by quantum fluctuationshas unveiled states with exotic fractional excitations and, frequently, with topological order [1]. The case of the geometrically frustrated two-dimensional (2D) Heisenberg quantum kagome antiferromagnet (QKA) is a prime example [1,2]. For this model, after numerous theoretical studies proposed a multitude of different ground states, a gapped SL state has been lately advocated [3-5] and also experimentally indicated [6]. However, its exact nature, as well as its topological classification, remains unclear [4,5,7-9]. Moreover, fierce competition seems to exist among candidate SL ground states of the QKA $[10,11]$. Therefore, the true ground state could be very sensitive to perturbations, leaving room for unconventional instabilities such as nontrivial symmetry breaking [12] or topological ordering. Recently, an instability of this kind has indeed been observed experimentally, but on the related 2D triangular lattice [13].

To explore possible instabilities of the kagome lattice, we focus on $\mathrm{ZnCu}_{3}(\mathrm{OH})_{6} \mathrm{SO}_{4}$, a compound with intriguing magnetic properties [14] that has recently joined a small number of QKA systems with a possible SL ground state [1518]. Notwithstanding theoretical predictions claiming the contrary, the majority of these systems appears to be gapless $[14,17,18]$. Therefore, in-depth experimental studies of new QKA candidates that reveal common features as well as subtle differences among the studied materials are very important. One particularly relevant issue is the role of impurities in the magnetism of QKAs [19-23]. This has been experimentally extensively studied in herbertsmithite [24-27], $\mathrm{ZnCu}_{3}(\mathrm{OH})_{6} \mathrm{Cl}_{2}$, which is recognized as the best realization of the QKA model so far, despite substantial (5-10\%) $\mathrm{Cu}-\mathrm{Zn}$ intersite disorder [28]. In this compound, such impurities were found to dominate bulk magnetic excitations at low temperatures and low energies $(\lesssim 0.8 \mathrm{meV})$ [26,27]. The

\footnotetext{
*andrej.zorko@ijs.si
}

compound investigated here closely resembles herbertsmithite in the degree of the $\mathrm{Cu}-\mathrm{Zn}$ intersite disorder (6-9\%; Ref. [14]). However, the two crystal structures are notably different, as in $\mathrm{ZnCu}_{3}(\mathrm{OH})_{6} \mathrm{SO}_{4}$ the $\mathrm{Zn}^{2+}$ site lies within the kagome planes, while it is positioned between the kagome layers in herbertsmithite. Consequently, $\mathrm{ZnCu}_{3}(\mathrm{OH})_{6} \mathrm{SO}_{4}$ features well-isolated 2D kagome planes, where three nonequivalent $\mathrm{Cu}^{2+}$ sites are coupled with an average intrakagome exchange interaction of $J=65 \mathrm{~K}$ [14]. Bulk susceptibility $\left(\chi_{\mathrm{b}}\right)$ and heat capacity $\left(C_{\mathrm{p}}\right)$ measurements failed to detect any sign of magnetic ordering down to temperatures $T$ three orders of magnitude below $J$ [14]. Moreover, a $T$-independent intrinsic kagome susceptibility $\chi_{\mathrm{k}}$ [see Fig. 1(b)] and a linearly increasing magnetic $C_{\mathrm{p}}$, which are both characteristic of spinon excitations with a pseudo-Fermi surface, were observed after subtraction of quasi-free-defect contributions from both quantities. Surprisingly, these two features signifying a gapless SL state were found in two $T$ regions, between 5 and $15 \mathrm{~K}$ (which we call SL1) as well as at $T<0.6 \mathrm{~K}$ (SL2) [14]. Such an intricate, two-state SL behavior, which appears unique among QKA representatives, clearly requires further verification and deeper understanding.

Here we provide a unique experimental viewpoint on the stability of the SL states in $\mathrm{ZnCu}_{3}(\mathrm{OH})_{6} \mathrm{SO}_{4}$ that is related to the general issue of the QKA ground state, by combining inelastic neutron scattering (INS) with two localprobe spectroscopic techniques employing different coupling mechanisms, nuclear magnetic resonance (NMR) and muon spin relaxation $(\mu \mathrm{SR})$. The temperature- and field-dependent NMR and $\mu \mathrm{SR}$ spin-lattice relaxation $\left(1 / T_{1}\right)$ measurements, supported by INS experiments that yield complementary information on the spatial and temporal spin correlations, expose various distinct fluctuating regimes. $\mu \mathrm{SR}$, performed down to $21 \mathrm{mK}$, gives evidence of a fluctuating SL ground state, while NMR discloses another state with characteristics of a gapless SL at higher temperatures. In combination with INS, the NMR $1 / T_{1}$ measurements reveal that this high- $T$ SL state is followed by a critical region at even higher temperatures, where the scaling of the dynamical susceptibility 

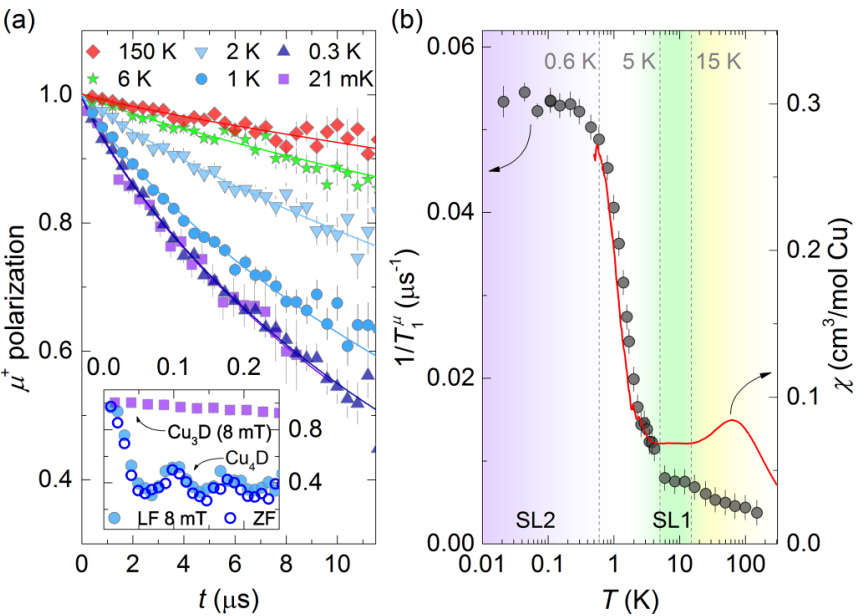

FIG. 1. (a) The $\mu^{+}$polarization in $\mathrm{ZnCu}_{3}(\mathrm{OD})_{6} \mathrm{SO}_{4}\left(\mathrm{Cu}_{3} \mathrm{D}\right)$ in a LF of $8 \mathrm{mT}$ (symbols) and the corresponding fits from a stretchedexponential model (lines; see text for details). In the inset the low- $T$ data of $\mathrm{Cu}_{3} \mathrm{D}$ are compared to $1.6 \mathrm{~K}$ data of $\mathrm{Cu}_{4}(\mathrm{OD})_{6} \mathrm{SO}_{4}$ $\left(\mathrm{Cu}_{4} \mathrm{D}\right)$ in the same LF and in zero field. (b) The longitudinal muon relaxation rate $1 / T_{1}^{\mu}$ compared to the intrinsic kagome susceptibility $\chi_{\mathrm{k}}$ measured at $0.1 \mathrm{~T}$ [14]. The two different SL regimes are denoted by SL1 and SL2.

with $\omega / T$ is obeyed over several decades in frequency $\omega$. This complex sequence of correlated electronic states suggesting SL instabilities is likely a more general feature of frustrated antiferromagnets.

$\mu \mathrm{SR}$ is a particularly powerful technique for detecting even the smallest magnetic fields $B_{\mu}$, of either nuclear or electronic origin, at the muon stopping site and for quantifying their dynamics [29]. The measurements were performed on the general Purpose Surface-Muon (GPS) and Low Temperature Facility (LTF) instruments, Paul Scherrer Institute (PSI), Switzerland, and on the EMU instrument at the ISIS Facility, Rutherford Appleton Laboratory, United Kingdom. The time decay of the muon spin polarization $P(t)$ is driven by the Zeeman coupling of the muon magnetic moment with $B_{\mu}$. It is presented in Fig. 1(a) for a longitudinal field (LF) of $8 \mathrm{mT}$, which isolates the relaxation due to fluctuating $\mathrm{Cu}^{2+}$ electronic moments [30]. The background-subtracted [30] data could be modeled with the stretched-exponential form $P_{\text {str }}(t)=\mathrm{e}^{-\left(t / T_{1}^{\mu}\right)^{\beta_{\mu}}}$, with the stretching exponent $\beta_{\mu}=0.86(6)$. As $T$ decreases from 150 $\mathrm{K}$, the muon relaxation rate $1 / T_{1}^{\mu}$ first increases gradually, but then gets substantially enhanced below $5 \mathrm{~K}$ [Fig. 1(b)]. Since the LF is small compared to the frequency $\omega_{e}$ of fluctuating $B_{\mu}\left(\gamma_{\mu} B_{\mathrm{LF}} \ll \omega_{\mathrm{e}} \sim k_{\mathrm{B}} J / \hbar ; \gamma_{\mu}=2 \pi \times 135.5 \mathrm{MHz} / \mathrm{T}, k_{\mathrm{B}}\right.$ and $\hbar$ denote the muon gyromagnetic ratio, the Boltzmann constant, and the reduced Planck constant, respectively), the standard exponential decay of the field autocorrelation function yields the muon relaxation rate $1 / T_{1}^{\mu} \propto\left(\gamma_{\mu} \Delta B_{\mu}\right)^{2} / \omega_{\mathrm{e}}$ [29]. The observed increase of $1 / T_{1}^{\mu}$ below $5 \mathrm{~K}$ is thus a sign of pronounced slowing down of electronic fluctuations and/or enlarged local-field distribution width $\Delta B_{\mu}$. It coincides with the low- $T$ increase of the intrinsic $\chi_{\mathrm{k}}$ [Fig. 1(b)]. $1 / T_{1}^{\mu}$ levels off below $\sim 0.6 \mathrm{~K}$ [Fig. 1(b)], signaling persistent dynamics of $B_{\mu}$ below this temperature. The low- $T$ increase of $1 / T_{1}^{\mu}$ and the relaxation plateau are both features common

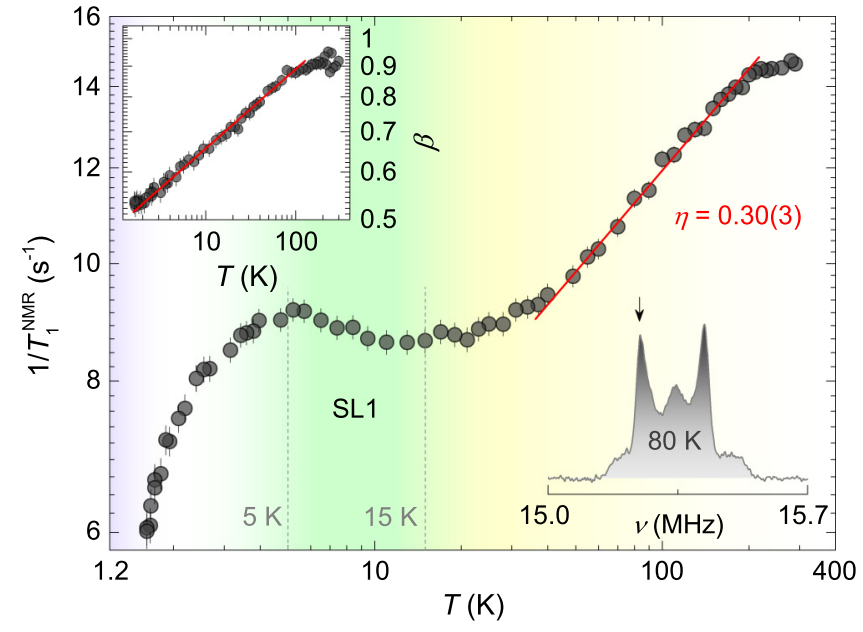

FIG. 2. The ${ }^{2} \mathrm{D} N M R$ relaxation rate $1 / T_{1}^{\mathrm{NMR}}$ measured at $2.35 \mathrm{~T}$ on the left spectral maximum of $\mathrm{ZnCu}_{3}(\mathrm{OD})_{6} \mathrm{SO}_{4}$ (arrow in the lower inset, showing the NMR spectrum at $80 \mathrm{~K}$ ), obtained from the model of magnetic relaxation (see the text). The straight line points out the region where $1 / T_{1} \propto T^{\eta}$. The upper inset depicts the $T$ dependence of the stretching exponent $\beta$, also showing a power-law dependence below $100 \mathrm{~K}$.

to different kagome antiferromagnets [16-18,31,32]. The unchanged shape of $P(t)$ unquestionably reveals that any kind of electronic freezing is absent in $\mathrm{ZnCu}_{3}(\mathrm{OD})_{6} \mathrm{SO}_{4}$ at least down to $21 \mathrm{mK}$, that is $T / J \geqslant 1 / 3000$. This stands in sharp contrast to the related magnetically ordered $\mathrm{Cu}_{4}(\mathrm{OD})_{6} \mathrm{SO}_{4}$, where an oscillatory $P(t)$ is observed below its Néel temperature of $7.5 \mathrm{~K}$ [33]. In that compound the first local minimum occurs at $t=\frac{\pi}{\gamma_{\mu} B_{\mu}}=0.062 \mu \mathrm{s}$ at $1.6 \mathrm{~K}$ [inset in Fig. 1(a)] and corresponds to a static $B_{\mu}=60 \mathrm{mT}$.

Further insight into the spin fluctuations in $\mathrm{ZnCu}_{3}(\mathrm{OD})_{6} \mathrm{SO}_{4}$ is provided by ${ }^{2} \mathrm{D}$ NMR spin-lattice relaxation. These measurements were performed on the lower-frequency spectral maximum (lower inset in Fig. 2; details on static NMR properties are given in Ref. [30]). The relaxation rate $1 / T_{1}^{\mathrm{NMR}}$ is determined by fluctuating local magnetic fields, as the experimental magnetization inversion-recovery curves are explained well with the magnetic-relaxation model for spin-1 nuclei [34], $M(t)=M_{0}\left[1-(1+s)\left(\frac{1}{4} \mathrm{e}^{-\left(t / T_{1}\right)^{\beta}}+\frac{3}{4} \mathrm{e}^{-\left(3 t / T_{1}\right)^{\beta}}\right)\right]$, with $s<1$ due to imperfect inversion of broad NMR lines and with $\beta$ denoting the stretching exponent. $1 / T_{1}^{\mathrm{NMR}}$ is set by spin fluctuations at the Larmor frequency $\omega_{\mathrm{L}}$,

$$
\frac{1}{T_{1}^{\mathrm{NMR}}} \propto \gamma^{2} T \sum_{\mathbf{Q}}\left|A_{\mathbf{Q}}\right|^{2} \frac{\chi^{\prime \prime}\left(\mathbf{Q}, \omega_{\mathrm{L}}\right)}{\omega_{\mathrm{L}}},
$$

where $\chi^{\prime \prime}\left(\mathbf{Q}, \omega_{\mathrm{L}}\right)$ stands for the wave-vector dependent dynamical susceptibility and $A_{\mathbf{Q}}$ is the coupling between the ${ }^{2} \mathrm{D}$ nuclear and $\mathrm{Cu}^{2+}$ electronic moments. In a paramagnetic regime a temperature-independent relaxation rate is expected [35]. In $\mathrm{ZnCu}_{3}(\mathrm{OD})_{6} \mathrm{SO}_{4}$, deviations from such a behavior are already found at room $T$ and are substantially enhanced below $200 \mathrm{~K}$ (Fig. 2). The observed decrease of $1 / T_{1}^{\mathrm{NMR}}$ is a robust sign of developing spin correlations and is accompanied by a significant increase of the $T_{1}$-distribution width, evidenced by $\beta$ decreasing from $0.93(3)$ at $300 \mathrm{~K}$ to 
$0.53(3)$ at $1.6 \mathrm{~K}$ (upper inset in Fig. 2). Such a $T$-dependent broadening of the $T_{1}$ distribution is often observed in frustrated antiferromagnets [13,36-38] and is generally recognized as a fingerprint of correlated disordered states. On the contrary, a trivial reduction of $\beta$ imposed by impurities that lead to a distribution of $A_{\mathbf{Q}}$ 's is $T$ independent [38].

Between 200 and $40 \mathrm{~K}$, we find $1 / T_{1}^{\mathrm{NMR}} \propto T^{\eta}$ with $\eta=0.30(2)$. A similar power-law behavior was previously observed in a number of 2D SL candidates, where it was attributed to gapless excitations [39-43]. $1 / T_{1}^{\mathrm{NMR}} \propto T^{\eta}$ is theoretically expected for a Dirac U(1) SL on the kagome lattice $[41,44]$; however, for such a state the theory predicts $\chi \propto T$ and $C_{\mathrm{p}}^{\mathrm{ZF}} \propto T^{2}$, which both contradict the experimental observations in $\mathrm{ZnCu}_{3}(\mathrm{OH})_{6} \mathrm{SO}_{4}$ [14]. Alternatively, a gapped $Z_{2}$ SL in the quantum-critical region close to an antiferromagnetically ordered state of a triangular lattice also exhibits power-law NMR relaxation [45]. Moreover, $1 / T_{1}^{\mathrm{NMR}} \propto T^{\eta}$ is well established in one-dimensional (1D) spin systems [46]. There, it is considered a hallmark of quantum criticality emerging at $T \lesssim J$ and $\eta$ ranges from positive to negative values as the magnetic field is tuned across the quantum critical region. With the universality of quantum-critical spin fluctuations [47], the observed power-law dependence in $\mathrm{ZnCu}_{3}(\mathrm{OD})_{6} \mathrm{SO}_{4}$ may well be a signature of criticality. In such a case, the energy scale is determined solely by temperature and is independent of the microscopic characteristics of the Hamiltonian. Therefore, one expects a scaling of the form $\chi^{\prime \prime}(\omega) \propto|\omega|^{v} F\left(\hbar \omega / k_{\mathrm{B}} T\right)[47]$.

In order to verify this interesting possibility in $\mathrm{ZnCu}_{3}(\mathrm{OD})_{6} \mathrm{SO}_{4}$, we performed powder INS measurements. The data were collected on the FOCUS instrument at PSI, Switzerland. After using an inovative method for the subtraction of background we developed in Ref. [30], the magnetic contribution to the scattering intensity, $S(\omega)$, was obtained by $Q$ integration $\left(0.35 \AA^{-1} \leqslant Q \leqslant 1.4 \AA^{-1}\right)$. From $S(\omega)$ the imaginary part of the dynamical susceptibility, $\chi^{\prime \prime}(\omega)=$ $S(\omega)\left[1-\mathrm{e}^{-\hbar \omega / k_{\mathrm{B}} T}\right]$, was derived (Fig. 3). Interestingly, above
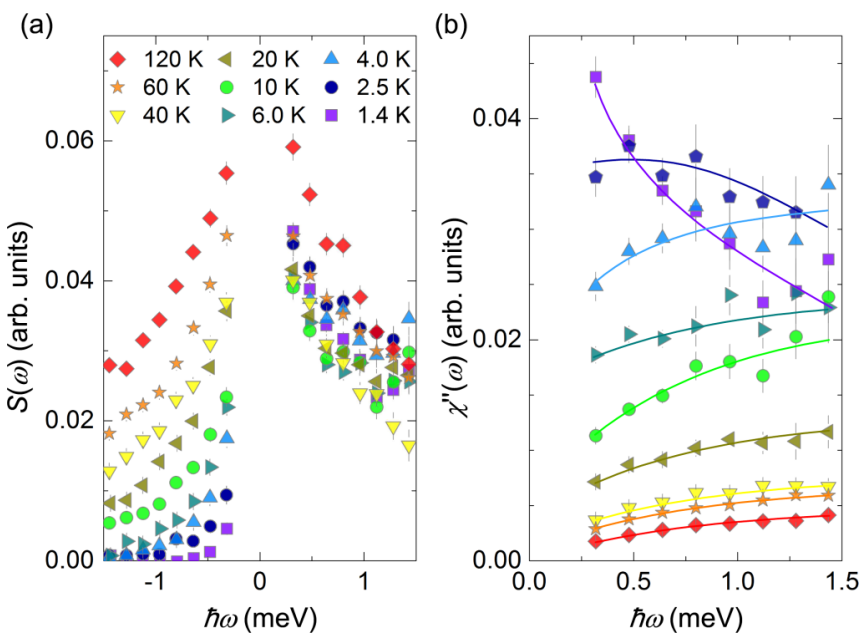

FIG. 3. (a) The magnetic contribution to the neutron-scattering intensity, $S(\omega)$, in $\mathrm{ZnCu}_{3}(\mathrm{OD})_{6} \mathrm{SO}_{4}$, where $Q$ integration was performed over wave vectors $0.35 \AA^{-1} \leqslant Q \leqslant 1.4 \AA^{-1}$, and (b) the related dynamical susceptibility $\chi^{\prime \prime}(\omega)$. Solid lines are a guide to the eye.

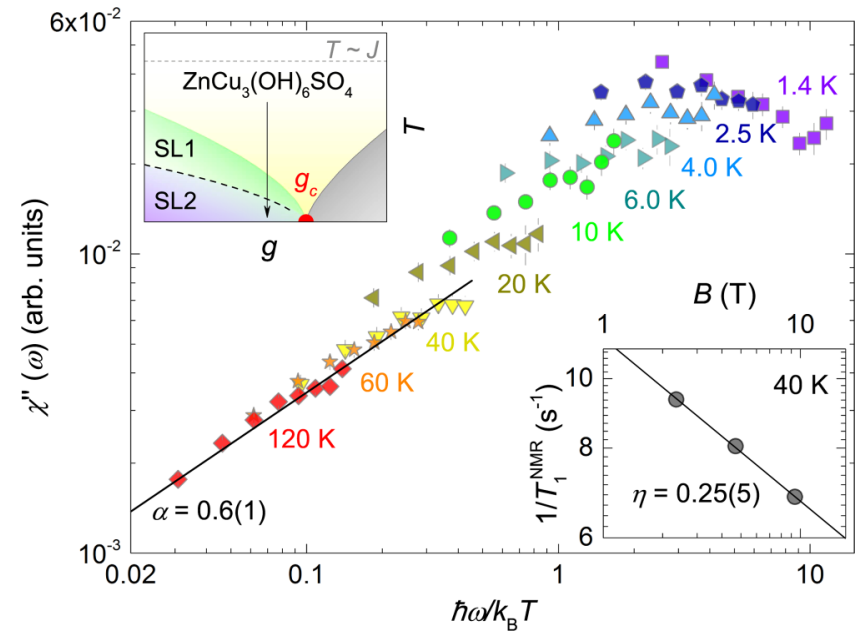

FIG. 4. Scaling of the dynamical susceptibility with the reduced energy following a power law $\chi^{\prime \prime} \propto\left(\hbar \omega / k_{\mathrm{B}} T\right)^{\alpha}$ above $\sim 40 \mathrm{~K}$. Lower inset: the $1 / T_{1}^{\mathrm{NMR}} \propto B^{-\eta}$ field dependence of the ${ }^{2} \mathrm{D}$ NMR relaxation rate at $40 \mathrm{~K}$. Upper inset: a schematic $T-g$ phase diagram with a quantum critical point between SL and some other phase at the critical tuning parameter value $g_{c}$ and the associated quantum critical region (yellow). The SL instability that leads to two distinct SL phases is indicated by the dashed line.

$40 \mathrm{~K}, \chi^{\prime \prime}(\omega)$ was found to scale with reduced energy as $\chi^{\prime \prime}(\omega) \propto\left(\hbar \omega / k_{\mathrm{B}} T\right)^{\alpha}$, where $\alpha=0.6(1)$ (Fig. 4). Such a scaling law leads, according to Eq. (1), to $1 / T_{1}^{\mathrm{NMR}} \propto(T / \omega)^{\eta}$ with $\eta=1-\alpha=0.4(1)$. This is in good agreement with $\eta=0.30$ (2) obtained from the NMR data. Moreover, the predicted power-law field dependence $1 / T_{1}^{\mathrm{NMR}} \propto \omega^{-\eta} \propto B^{-\eta}$ is also observed experimentally, with $\eta=0.25(5)$ (lower inset in Fig. 4), thus providing additional verification of the proposed criticality. We note that similarly $B$-dependent $1 / T_{1}^{\mathrm{NMR}}$ is found in quantum-critical 1D systems [46], while impurities would typically yield a more pronounced dependence, with $1 / T_{1}^{\mathrm{NMR}} \propto B^{-1}$ [48]. The astonishing feature that emerges when combining NMR and INS results on $\mathrm{ZnCu}_{3}(\mathrm{OD})_{6} \mathrm{SO}_{4}$ in the $T$ range $40-200 \mathrm{~K}$ is that the $\chi^{\prime \prime}(\omega)$ scaling observed in the INS data in the energy range $0.3-2 \mathrm{meV}$ extends to several orders of magnitude lower energies, as $\hbar \omega_{\mathrm{L}}=0.06 \mu \mathrm{eV}$ in NMR. Such a broad range of universal fluctuation indeed appears to be a fingerprint of criticality. Scale-free intrinsic kagome-lattice correlations that persist to high $T$ at high energies were also observed in herbertsmithite [24]. There, the impurity contribution to $\chi^{\prime \prime}(\omega)$ is limited to $T<10 \mathrm{~K}$ [26] and $\hbar \omega<0.8 \mathrm{meV}$ [27]. As the effective low- $T$ Curie-Weiss temperature of the impurity spins is approximately $-1 \mathrm{~K}$ in $\mathrm{ZnCu}_{3}(\mathrm{OH})_{6} \mathrm{SO}_{4}$ [14] as well as in herbertsmithite [28] and the amount of impurities in both compounds is very similar, we exclude any role of quasifree impurities in the scaling presented in Fig. 4. On the other hand, any observed scaling at low $T$ and $\omega$ [49] could be biased by impurity contributions.

Below $40 \mathrm{~K}$, the high- $T$ scaling of the dynamical susceptibility is not valid, as the $\chi^{\prime \prime}(\omega, T)$ data sets from different temperatures no longer overlap (Fig. 4). At the same temperature, the power-law dependence of $1 / T_{1}^{\mathrm{NMR}}$ (Fig. 2) starts to flatten. It exhibits a shallow minimum at $\sim 15 \mathrm{~K}$ and a maximum at 
$\sim 5 \mathrm{~K}$ (Fig. 2). In sharp contrast, $\beta$ evolves monotonically, proving that such behavior of $1 / T_{1}^{\mathrm{NMR}}$ is intrinsic. The $1 / T_{1}^{\mathrm{NMR}}(T)$ dependence remains qualitatively unchanged even if we use a fixed $\beta$ to fit the data. The essentially $T$-independent region of $1 / T_{1}^{\mathrm{NMR}}$ between the shallow minimum and the maximum coincides with the temperature-independent region of intrinsic susceptibility [Fig. 1(b)] and the linearly dependent heat capacity [14]. It can thus be attributed to a correlated SL state of the system below $\sim 15 \mathrm{~K}$. We note that the flattening of $1 / T_{1}^{\mathrm{NMR}}$ below $40 \mathrm{~K}$ cannot be attributed to weakly interacting impurities, as the measured NMR-line shift is driven by intrinsic kagome spins at least down to $10 \mathrm{~K}$ [30], which differs from the situation encountered in herbertsmithite [50].

The dynamical properties of the magnetic state in $\mathrm{ZnCu}_{3}(\mathrm{OD})_{6} \mathrm{SO}_{4}$ change again below $5 \mathrm{~K}$, as a steady decrease of $1 / T_{1}^{\mathrm{NMR}}$ is observed once more upon cooling (Fig. 2). Such a rich $T$ dependence clearly reflects multiple diverse states that the system goes through with changing temperature. At the same time, no anomalies are observed in the static properties of the NMR spectra [30]. Interestingly, a qualitatively similar behavior of $1 / T_{1}^{\mathrm{NMR}}$ with two different power-law correlated regimes and a flat $T$ region in between was found in organic triangular-lattice SL candidates $[13,36]$. In $\mathrm{EtMe}_{3} \mathrm{Sb}\left[\mathrm{Pd}\left(\mathrm{dmit}_{2}\right)\right]_{2}$, such an intricate behavior was attributed to a symmetry breaking and/or a topological instability of a gapless SL phase [13]. Based on our $1 / T_{1}^{\mathrm{NMR}}$ data, we propose that a similar crossover between different SL1 and SL2 phases is at work in $\mathrm{ZnCu}_{3}(\mathrm{OD})_{6} \mathrm{SO}_{4}$.

This crossover is further witnessed by the muon relaxation rate $1 / T_{1}^{\mu}$ [Fig. 1(b)], which suddenly starts to increase below $5 \mathrm{~K}$, after exhibiting a very gentle $T$ dependence between 15 and $5 \mathrm{~K}$, being in line with the high- $T$ SL1 phase. We note that $\mu$ SR yields a somewhat different, more indirect viewpoint on the spin dynamics than NMR. Namely, the $\mu$ SR experiment was performed in a small external field $\left(B_{\mathrm{LF}} \ll B_{\mu}\right)$, and therefore Eq. (1), which is derived using time-dependent perturbation theory in the limit of large external fields, is not directly applicable [51]. Moreover, in $\mu$ SR, contrary to NMR, the long-range dipolar interactions typically overshadow the contact hyperfine interaction in magnetic insulators [29]. Therefore, in $\mathrm{ZnCu}_{3}(\mathrm{OD})_{6} \mathrm{SO}_{4}$, the effective spatial averaging of the dipolar interaction makes the muon relaxation due to the relatively dense impurity spins dominant at low temperatures, where $\chi_{\mathrm{k}} \ll \chi_{\mathrm{b}}$ [14]. The same behavior was indeed observed in herbertsmithite, where the low- $T 1 / T_{1}^{\mu}$ scaled linearly with the quantity of $\mathrm{Cu}^{2+}$ impurities on the $\mathrm{Zn}$ site [31]. The increase of $1 / T_{1}^{\mu}$ below $5 \mathrm{~K}$ can in our case then be ascribed to evolving correlations between impurity and intrinsic kagome spins. Judging from the relaxation plateau, these correlations saturate within the low- $T$ SL2 state below $0.6 \mathrm{~K}$, suggesting that an effective impurity coupling is established. Because of a finite $T$-independent $\chi_{\mathrm{k}}$ and linear $C_{\mathrm{p}}(T)$ below $0.6 \mathrm{~K}$ [14] the SL2 state appears to be gapless, with any gap smaller than $J / 3000$ as evidenced by persisting muon relaxation observed down to at least $21 \mathrm{mK}$.
From a theoretical standpoint, a gapped SL ground state, possibly of $Z_{2}$ type, is expected for the isotropic Heisenberg QKA model [3-5]. The weight of magnetic excitations could, however, be considerably shifted towards zero energy due to a proximate valence bond solid (VBS) state [8], as was proposed from experimental observations on herbertsmithite [25]. Alternatively, quantum criticality between the $Z_{2}$ SL and an antiferromagnetically ordered state can be induced by the Dzyaloshinskii-Moriya interaction [52,53], which is known to be sizable in other $\mathrm{Cu}$-based QKA representatives $[54,55]$ and could also justify a gapless state [56]. Such a magnetic anisotropy that acts as a tuning parameter, being slightly off its critical value $g_{\mathrm{c}}$, may explain the scaling behavior observed in $\mathrm{ZnCu}_{3}(\mathrm{OD})_{6} \mathrm{SO}_{4}$ around $T \sim J$ and the selection of the gapless high- $T$ SL1 state around $15 \mathrm{~K}(T / J \sim 0.23)$, in the sense of the schematic phase diagram shown in Fig. 4. It is intriguing that the crossover from this state into the SL2 state is stabilized at even lower temperatures, that is below $0.6 \mathrm{~K}(T / J \sim 0.01)$.

With two clearly distinguishable SL regimes the investigated compound is unique among QKAs. The crossover between the two regimes observed in NMR and $\mu \mathrm{SR}$ relaxation is not reflected in the static NMR properties. Hence, the distinction between the two SL states must originate from spin excitations. Therefore, this crossover may well be of a topological character or due to nontrivial symmetry breaking. In fact, for gapless SL states with a spinon pseudo-Fermi surface various spinon-pairing instabilities can be expected at low temperatures [12,57,58]. Alternatively, impurities due to the $\mathrm{Cu}-\mathrm{Zn}$ intersite disorder inherent to the investigated system that are strongly coupled to the kagome lattice could pin spinons at low temperatures and thus provide a spinon-instability mechanism. While such a premise calls for further theoretical investigations, we note that the intriguing instability of the SL observed in $\mathrm{ZnCu}_{3}(\mathrm{OH})_{6} \mathrm{SO}_{4}$ appears to be a more general feature of geometrically frustrated antiferromagnets, common to $2 \mathrm{D}$ kagome and organic triangular lattices [13] that possess a much smaller quantity of impurities, and possibly even to the 3D hyperkagome lattice [59]. We expect that such an emergent behavior of SLs is a fingerprint of particular type of low-energy excitations, common to various geometrically frustrated spin lattices.

We thank P. Mendels and F. Bert for fruitful discussions. The financial support of the Slovenian Research Agency (Program No. P1-0125) and the Swiss National Science Foundation (SCOPES Project IZ73Z0_152734/1 and Grant No. 200021 140862) is acknowledged. Q.M.Z. was supported by the NSF of China and the Ministry of Science and Technology of China (973 Projects 2012CB921701). The $\mu \mathrm{SR}$ project has received funding from the European Union's Seventh Framework Programme for research, technological development, and demonstration under the NMI3-II Grant No. 283883. Neutron scattering experiments were performed at the Swiss spallation neutron source SINQ, Paul Scherrer Institute, Villigen, Switzerland. We thank C. Baines for his technical assistance at the Paul Scherrer Institute.
[1] L. Balents, Spin liquids in frustrated magnets, Nature (London) 464, 199 (2010).
[2] S. Sachdev, Kagomé- and triangular-lattice Heisenberg antiferromagnets: Ordering from quantum fluctuations and quantum- 
disordered ground states with unconfined bosonic spinons, Phys. Rev. B 45, 12377 (1992).

[3] S. Yan, D. A. Huse, and S. R. White, Spin-liquid ground state of the $S=1 / 2$ kagome Heisenberg antiferromagnet, Science 332, 1173 (2011).

[4] S. Depenbrock, I. P. McCulloch, and U. Schollwöck, Nature of the Spin-Liquid Ground State of the $S=1 / 2$ Heisenberg Model on the Kagome Lattice, Phys. Rev. Lett. 109, 067201 (2012).

[5] H.-C. Jiang, Z. Wang, and L. Balents, Identifying topological order by entanglement entropy, Nat. Phys. 8, 902 (2012).

[6] M. Fu, T. Imai, T.-H. Han, and Y. S. Lee, Evidence for a gapped spin-liquid ground state in a kagome Heisenberg antiferromagnet, Science 350, 655 (2015).

[7] Y.-C. He and Y. Chen, Distinct Spin Liquids and their Transitions in Spin-1/2 XXZ Kagome Antiferromagnets, Phys. Rev. Lett. 114, 037201 (2015).

[8] M. Punk, D. Chowdhury, and S. Sachdev, Topological excitations and the dynamic structure factor of spin liquids on the kagome lattice, Nat. Phys. 10, 289 (2014).

[9] M. P. Zaletel and A. Vishwanath, Constraints on Topological Order in Mott Insulators, Phys. Rev. Lett. 114, 077201 (2015).

[10] Y. Iqbal, F. Becca, S. Sorella, and D. Poilblanc, Gapless spinliquid phase in the kagome spin-1/2 Heisenberg antiferromagnet, Phys. Rev. B 87, 060405 (2013).

[11] Y. Iqbal, D. Poilblanc, and F. Becca, Vanishing spin gap in a competing spin-liquid phase in the kagome Heisenberg antiferromagnet, Phys. Rev. B 89, 020407 (2014).

[12] B. K. Clark, J. M. Kinder, E. Neuscamman, G. K.-L. Chan, and M. J. Lawler, Striped Spin Liquid Crystal Ground State Instability of Kagome Antiferromagnets, Phys. Rev. Lett. 111, 187205 (2013).

[13] T. Itou, A. Oyamada, S. Maegawa, and R. Kato, Instability of a quantum spin liquid in an organic triangular-lattice antiferromagnet, Nat. Phys. 6, 673 (2010).

[14] Y. Li, B. Pan, S. Li, W. Tong, L. Ling, Z. Yang, J. Wang, Z. Chen, $\mathrm{Z}$. Wu, and Q. M. Zhang, Gapless quantum spin liquid in the $S=1 / 2$ anisotropic kagome antiferromagnet $\mathrm{ZnCu}_{3}(\mathrm{OH})_{6} \mathrm{SO}_{4}$, New J. Phys. 16, 093011 (2014).

[15] J. S. Helton, K. Matan, M. P. Shores, E. A. Nytko, B. M. Bartlett, Y. Yoshida, Y. Takano, A. Suslov, Y. Qiu, J.-H. Chung, D. G. Nocera, and Y. S. Lee, Spin Dynamics of the Spin-1/2 Kagome Lattice Antiferromagnet $\mathrm{ZnCu}_{3}(\mathrm{OH})_{6} \mathrm{Cl}_{2}$, Phys. Rev. Lett. 98, 107204 (2007).

[16] P. Mendels, F. Bert, M. A. De Vries, A. Olariu, A. Harrison, F. Duc, J. C. Trombe, J. S. Lord, A. Amato, and C. Baines, Quantum Magnetism in the Paratacamite Family: Towards an Ideal Kagomé Lattice, Phys. Rev. Lett. 98, 077204 (2007).

[17] B. Fåk, E. Kermarrec, L. Messio, B. Bernu, C. Lhuillier, F. Bert, P. Mendels, B. Koteswararao, F. Bouquet, J. Ollivier, A. D. Hillier, A. Amato, R. H. Colman, and A. S. Wills, Kapellasite: A Kagome Quantum Spin Liquid with Competing Interactions, Phys. Rev. Lett. 109, 037208 (2012).

[18] L. Clark, J. C. Orain, F. Bert, M. A. De Vries, F. H. Aidoudi, R. E. Morris, P. Lightfoot, J. S. Lord, M. T. F. Telling, P. Bonville, J. P. Attfield, P. Mendels, and A. Harrison, Gapless Spin Liquid Ground State in the $S=1 / 2$ Vanadium Oxyfluoride Kagome Antiferromagnet $\left[\mathrm{NH}_{4}\right]_{2}\left[\mathrm{C}_{7} \mathrm{H}_{14} \mathrm{~N}\right]\left[\mathrm{V}_{7} \mathrm{O}_{6} \mathrm{~F}_{18}\right]$, Phys. Rev. Lett. 110, 207208 (2013).
[19] S. Dommange, M. Mambrini, B. Normand, and F. Mila, Static impurities in the $S=1 / 2$ kagome lattice: Dimer freezing and mutual repulsion, Phys. Rev. B 68, 224416 (2003).

[20] I. Rousochatzakis, S. R. Manmana, A. M. Läuchli, B. Normand, and F. Mila, Dzyaloshinskii-Moriya anisotropy and nonmagnetic impurities in the $s=\frac{1}{2}$ kagome system $\mathrm{ZnCu}_{3}(\mathrm{OH})_{6} \mathrm{Cl}_{2}$, Phys. Rev. B 79, 214415 (2009).

[21] R. R. P. Singh, Valence Bond Glass Phase in Dilute Kagome Antiferromagnets, Phys. Rev. Lett. 104, 177203 (2010).

[22] H. Kawamura, K. Watanabe, and T. Shimokawa, Quantum spin-liquid behavior in the spin-1/2 random-bond Heisenberg antiferromagnet on the kagome lattice, J. Phys. Soc. Jpn. 83, 103704 (2014).

[23] T. Shimokawa, K. Watanabe, and H. Kawamura, Static and dynamical spin correlations of the $S=1 / 2$ random-bond antiferromagnetic Heisenberg model on the triangular and kagome lattices, Phys. Rev. B 92, 134407 (2015).

[24] M. A. de Vries, J. R. Stewart, P. P. Deen, J. O. Piatek, G. J. Nilsen, H. M. Rønnow, and A. Harrison, Scale-Free Antiferromagnetic Fluctuations in the $s=1 / 2$ Kagome Antiferromagnet Herbertsmithite, Phys. Rev. Lett. 103, 237201 (2009).

[25] T.-H. Han, J. S. Helton, S. Chu, D. G. Nocera, J. A. RodriguezRivera, C. Broholm, and Y. S. Lee, Fractionalized excitations in the spin-liquid state of a kagome-lattice antiferromagnet, Nature (London) 492, 406 (2012).

[26] G. J. Nilsen, M. A. de Vries, J. R. Stewart, A. Harrison, and H. M. Rønnow, Low-energy spin dynamics of the $s=1 / 2$ kagome system herbertsmithite, J. Phys. Condens. Matter 25, 106001 (2013).

[27] T.-H. Han, M. R. Norman, J.-J. Wen, J. A. Rodriguez-Rivera, J. S. Helton, C. Broholm, and Y. S. Lee, Correlated impurities and intrinsic spin liquid physics in the kagome material Herbertsmithite, arXiv:1512.06807 (unpublished).

[28] P. Mendels and F. Bert, Quantum kagome antiferromagnet $\mathrm{ZnCu}_{3}(\mathrm{OH})_{6} \mathrm{Cl}_{2}$, J. Phys. Soc. Jpn. 79, 011001 (2010).

[29] A. Yaouanc and P. D. De Réotier, Muon Spin Rotation, Relaxation, and Resonance: Applications to Condensed Matter (Oxford University Press, Oxford, 2011).

[30] See Supplemental Material at http://link.aps.org/supplemental/ 10.1103/PhysRevB.93.060405 for details on the $\mu$ SR experiment, the ${ }^{2} \mathrm{D}$ NMR spectral analysis, and the INS background determination.

[31] E. Kermarrec, P. Mendels, F. Bert, R. H. Colman, A. S Wills, P. Strobel, P. Bonville, A. Hillier, and A. Amato, Spinliquid ground state in the frustrated kagome antiferromagnet $\mathrm{MgCu}_{3}(\mathrm{OH})_{6} \mathrm{Cl}_{2}$, Phys. Rev. B 84, 100401 (2011).

[32] A. Zorko, F. Bert, P. Mendels, P. Bordet, P. Lejay, and J. Robert, Easy-Axis Kagome Antiferromagnet: Local-Probe Study of $\mathrm{Nd}_{3} \mathrm{Ga}_{5} \mathrm{SiO}_{14}$, Phys. Rev. Lett. 100, 147201 (2008).

[33] S. Vilminot, M. Richard-Plouet, G. André, D. Swierczynski, F. Bourée-Vigneron, and M. Kurmoo, Nuclear and magnetic structures and magnetic properties of synthetic broctite, $\mathrm{Cu}_{4}(\mathrm{OH})_{6} \mathrm{SO}_{4}$, Dalton Trans. 11, 1455 (2006).

[34] A. Suter, M. Mali, J. Roos, and D. Brinkmann, Mixed magnetic and quadrupolar relaxation in the presence of a dominant static Zeeman Hamiltonian, J. Phys. Condens. Matter 10, 5977 (1998).

[35] T. Moriya, Nuclear magnetic relaxation in antiferromagnetics, Prog. Theor. Phys. 16, 23 (1956).

[36] Y. Shimizu, K. Miyagawa, K. Kanoda, M. Maesato, and G. Saito, Emergence of inhomogeneous moments from spin liquid in the 
triangular-lattice Mott insulator $\kappa-(\mathrm{ET})_{2} \mathrm{Cu}_{2}(\mathrm{CN})_{3}$, Phys. Rev. B 73, 140407 (2006).

[37] J. A. Quilliam, F. Bert, R. H. Colman, D. Boldrin, A. S. Wills, and P. Mendels, Ground state and intrinsic susceptibility of the kagome antiferromagnet vesignieite as seen by ${ }^{5} 1 \mathrm{~V}$ NMR, Phys. Rev. B 84, 180401 (2011).

[38] E. Kermarrec, A. Zorko, F. Bert, R. H. Colman, B. Koteswararao, F. Bouquet, P. Bonville, A. Hillier, A. Amato, J. van Tol, A. Ozarowski, A. S. Wills, and P. Mendels, Spin dynamics and disorder effects in the $S=1 / 2$ kagome Heisenberg spin-liquid phase of kapellasite, Phys. Rev. B 90, 205103 (2014).

[39] Y. Shimizu, K. Miyagawa, K. Kanoda, M. Maesato, and G. Saito, Spin Liquid State in an Organic Mott Insulator with a Triangular Lattice, Phys. Rev. Lett. 91, 107001 (2003).

[40] T. Itou, A. Oyamada, S. Maegawa, M. Tamura, and R. Kato, Quantum spin liquid in the spin-1/2 triangular antiferromagnet $\mathrm{EtMe}_{3} \mathrm{Sb}\left[\mathrm{Pd}\left(\mathrm{dmit}_{2}\right)\right]_{2}$, Phys. Rev. B 77, 104413 (2008).

[41] T. Imai, E. A. Nytko, B. M. Bartlett, M. P. Shores, and D. G. Nocera, ${ }^{6} 3 \mathrm{Cu},{ }^{3} 5 \mathrm{Cl}$, and ${ }^{1} \mathrm{H}$ NMR in the $S=1 / 2$ Kagome Lattice $\mathrm{ZnCu}_{3}(\mathrm{OH})_{6} \mathrm{Cl}_{2}$, Phys. Rev. Lett. 100, 077203 (2008).

[42] A. Olariu, P. Mendels, F. Bert, F. Duc, J. C. Trombe, M. A. De Vries, and A. Harrison, ${ }^{1} 7 \mathrm{O}$ NMR Study of the Intrinsic Magnetic Susceptibility and Spin Dynamics of the Quantum Kagome Antiferromagnet $\mathrm{ZnCu}_{3}(\mathrm{OH})_{6} \mathrm{Cl}_{2}$, Phys. Rev. Lett. 100, 087202 (2008).

[43] T. Dey, A. V. Mahajan, R. Kumar, B. Koteswararao, F. C. Chou, A. A. Omrani, and H. M. Ronnow, Possible spin-orbit driven spin-liquid ground state in the double perovskite phase of $\mathrm{Ba}_{3} \mathrm{YIr}_{2} \mathrm{O}_{9}$, Phys. Rev. B 88, 134425 (2013).

[44] Y. Ran, M. Hermele, P. A. Lee, and X.-G. Wen, ProjectedWave-Function Study of the Spin-1/2 Heisenberg Model on the Kagomé Lattice, Phys. Rev. Lett. 98, 117205 (2007).

[45] Y. Qi, C. Xu, and S. Sachdev, Dynamics and Transport of the $Z_{2}$ Spin Liquid: Application to $\kappa-(\mathrm{ET})_{2} \mathrm{Cu}_{2}(\mathrm{CN})_{3}$, Phys. Rev. Lett. 102, 176401 (2009).

[46] S. Mukhopadhyay, M. Klanjšek, M. S. Grbić, R. Blinder, H. Mayaffre, C. Berthier, M. Horvatić, M. A. Continentino, A. Paduan-Filho, B. Chiari, and O. Piovesana, Quantum-Critical Spin Dynamics in Quasi-One-Dimensional Antiferromagnets, Phys. Rev. Lett. 109, 177206 (2012).

[47] S. Sachdev and J. Ye, Universal Quantum-Critical Dynamics of Two-Dimensional Antiferromagnets, Phys. Rev. Lett. 69, 2411 (1992).
[48] M. Klanjšek, D. Arčon, A. Sans, P. Adler, M. Jansen, and C. Felser, Phonon-Modulated Magnetic Interactions and Spin Tomonaga-Luttinger Liquid in the $p$-Orbital Antiferromagnet $\mathrm{CsO}_{2}$, Phys. Rev. Lett. 115, 057205 (2015).

[49] J. S. Helton, K. Matan, M. P. Shores, E. A. Nytko, B. M. Bartlett, Y. Qiu, D. G. Nocera, and Y. S. Lee, Dynamic Scaling in the Susceptibility of the Spin-1/2 Kagome Lattice Antiferromagnet Herbertsmithite, Phys. Rev. Lett. 104, 147201 (2010).

[50] T. Imai, M. Fu, T. H. Han, and Y. S. Lee, Local spin susceptibility of the $S=1 / 2$ kagome lattice in $\mathrm{ZnCu}_{3}(\mathrm{OD})_{6} \mathrm{Cl}_{2}$, Phys. Rev. B 84, 020411 (2011).

[51] C. Lacroix, P. Mendels, and F. Mila, eds., Introduction to Frustrated Magnetism: Materials, Experiments, Theory (Springer Verlag, Berlin, 2011).

[52] O. Cépas, C. M. Fong, P. W. Leung, and C. Lhuillier, Quantum phase transition induced by Dzyaloshinskii-Moriya interactions in the kagome antiferromagnet, Phys. Rev. B 78, 140405 (2008).

[53] Y. Huh, L. Fritz, and S. Sachdev, Quantum criticality of the kagome antiferromagnet with Dzyaloshinskii-Moriya interactions, Phys. Rev. B 81, 144432 (2010).

[54] A. Zorko, S. Nellutla, J. Van Tol, L. C. Brunel, F. Bert, F. Duc, J.-C. Trombe, M. A. De Vries, A. Harrison, and P. Mendels, Dzyaloshinsky-Moriya Anisotropy in the Spin-1/2 Kagome Compound $\mathrm{ZnCu}_{3}(\mathrm{OH})_{6} \mathrm{Cl}_{2}$, Phys. Rev. Lett. 101, 026405 (2008).

[55] A. Zorko, F. Bert, A. Ozarowski, J. van Tol, D. Boldrin, A. S. Wills, and P. Mendels, Dzyaloshinsky-Moriya interaction in vesignieite: A route to freezing in a quantum kagome antiferromagnet, Phys. Rev. B 88, 144419 (2013).

[56] T. F. Seman, C.-C. Chen, R. R. P. Singh, and M. van Veenendaal, The many faces of quantum kagome materials: Interplay of further-neighbour exchange and Dzyaloshinskii-Moriya interaction, arXiv:1508.01523 (2015).

[57] S.-S. Lee, P. A. Lee, and T. Senthil, Amperean Pairing Instability in the $U(1)$ Spin Liquid State with Fermi Surface and Application to $\kappa$-(BEDT-TTF $)_{2} \mathrm{Cu}_{2}(\mathrm{CN})_{3}$, Phys. Rev. Lett. 98, 067006 (2007).

[58] V. Galitski and Y. B. Kim, Spin-Triplet Pairing Instability of the Spinon Fermi Surface in a $U(1)$ Spin Liquid, Phys. Rev. Lett. 99, 266403 (2007).

[59] Y. Zhou, P. A. Lee, T.-K. Ng, and F.-C. Zhang, $\mathrm{Na}_{4} \mathrm{Ir}_{3} \mathrm{O}_{8}$ as a 3D Spin Liquid with Fermionic Spinons, Phys. Rev. Lett. 101, 197201 (2008). 